\title{
Fast Searching for CT Windows based on Golden Segmentation Algorithm
}

\author{
Hua jing, Li ning, Zhang ruilan, Liu yingjiu, Hu jiguang \\ Department of Biomedical Engineering, School of Medicine, Bei Hua University, Jilin city, Jilin province, 132013, China \\ E-mail: 4922515@qq.com
}

\begin{abstract}
Aims: To introduce the application of golden segmentation algorithm in CT window searching. Methods: The relationship between the appropriateness of $\mathrm{CT}$ windows and the quality of the image is a unimodal problem in mathematics. It is crucial for best displaying the CT image to find out the best window of unknown values (CT) range of tissues and organs. The criterion of the window level and window width was introduced in this article, to search for the window level and window width of the corresponding blur CT images and digital images with low resolution to the original data of human body by using golden segmentation algorithm program operations. Results: To find out the best window level and window width by using golden segmentation algorithm is time-used less, fast and good. Conclusion: It has certain advantages compared to other mature window technologies (such as double-window technique and multiple-window technique). This technique can be complement of $\mathrm{CT}$ window techniques and can provide reference for $\mathrm{CT}$ suppliers.

Index Terms - Golden segmentation algorithm, Window technologies, CT window techniques, CT double-Window technique
\end{abstract}

\section{Introduction}

The CT image directly established by the raw data which obtained by CT is blurred image of low resolution that cannot be used directly. The corresponding grayscale value of tissues and organs to this vague image is between a very narrow interval of grayscale among the grayscale (dark or all black to white or all bright), the corresponding grayscale difference (the difference between black and white) of image structure to each tissue or organs is so tiny that naked eyes cannot distinguish each structure of the image. So the very narrow interval of grayscale must be extended to the full grayscale (all black to all white), which means to extend (or to increase) the upper bound of this narrow grayscale to all white, to extend (or to compress) the lower bound of the grayscale to all black, thus the grayscale difference (the difference between black and white) of each structure in the image can be enlarged and each structure in the image can be seen by naked eyes. The technology which makes the realization of this process is called window technology. It can be said that the window technology is the technology which extends the grayscale difference (the difference between black and white) of each section in the image. The window image obtained by window technology is the CT image that can be directly used. The corresponding numerical range of different tissues and lesions differs, so that the corresponding range of grayscale which to be extended to different tissues or lesions is diverse, that is to say, different tissues and organs have different windows. The window contains the two important concepts of window level and window width. Window width is the width of the window, which means the numerical (CT value) range of tissues, organs and lesion, the maximum value (CT value) of this range is the maximum limit value CTmax of window width, the minimum value CTmin is a lower limit value, apparently, the theoretical window width should be the difference value of CTmax and CTmin, that is:

$$
\text { Window width }=\left(C T_{\text {max }}-C T_{\text {min }}\right)(\mathrm{HU})
$$

Window level is the median of this range, which is the arithmetic mean value of CTmax and CTmin, that is

$$
\text { Window level }=\frac{C T_{\max }+C T_{\min }}{2}(\mathrm{HU})
$$

For example, the CTmax of brain tissues is "+95HU", the CTmin is "-25HU", then we can determine that the window width of brain tissue is $120 \mathrm{HU}$, the window level is $35 \mathrm{HU}$.

From a mathematical point of view, the relationship between the values of window width and window level and CT image quality meets the unimodal curve (or curved surface), The corresponding data of the window (the window level and window width) to the curve (or curved surface) makes the CT image without losing any information (note: all values of the tissues and organs are within the window, there is no value outside the window; values outside the window do not participate in image display, so the corresponding image structure of the value outside the window is missing, and this is the lost information of the image), and has got the best display, the corresponding window level and window width of the peak is the most appropriate. To CT images of tissues and organs, from the point of the window, as long as there is deviation from the standard (peak) window level, both higher and lower, the image quality will drop (the value outside the window makes the lost of image information); to the window width, as long as there is deviation from the standard (peak) window width, both wider and narrower, the image quality will drop (with higher or lower contrast ratio). So to one CT image, the window selection is a unimodal curve (or curved surface). It is very essential in practice to find out the right window (peak) of the CT image to the unknown tissues and organs quickly.

\section{Text}

This article proposed the point that the peak or the window close to the peak can be quickly found by Golden section algorithm, and this makes the best or almost the best CT image. To human organism, the corresponding CT value is about $-1000 \mathrm{HU}$ to $+1000 \mathrm{HU}$, which has a total of approximate 
$2000 \mathrm{CT}$ values. To the $2000 \mathrm{CT}$ values, they had the corresponding 2000 different window levels from $-1000 \mathrm{HU}$ to $+1000 \mathrm{HU}$ and 2000 different window widths from $1 \mathrm{HU}$ to $2000 \mathrm{HU}$. Actually, the CT operators have a pretty good idea of the data (CT value) scope to most tissues and organs of human body, so they can quickly set the right window. If they are not familiar with the data (CT value) scope of some unknown tissues or organs, it will take some time for them to find out the right window (including the window level and window width). If the operators tried to find out the proper window by choosing different window levels and window widths one by one experimentally, in theory, they need to test 4000000 ( $=$ $2000 \times 2000)$ times. Even it takes 1 second to complete a test, day and night without rest, we need 46 days to finish looking for a suitable window of one CT image, and it is not feasible in practice.

Actually, the CT machine has a set of technologies such as double-windows, multiple-windows, as well as the window inside window technology. For example, the double-window technique can simultaneously display two different window images with different window levels and window widths. It is more convenient to compare the two images and to search with both windows, and to accelerate the search speed of the right window and its corresponding image. For the window search of tissues and organs (such as non-human species) and lesions with unknown value range, even using the double-window technique, it will take the magnitude of "minutes".

To make the proper window searching of the unknown tissues, organs and lesions, we only need several or a dozen attempts by using the golden section algorithm proposed in this paper, if the criterion is accurate and used skillfully, then only 1 second is needed to complete one segmentation search and the total spending is about 20 seconds to find out the right window.

The criteria will be introduced firstly, it can be summed up by "the high is black, the low is white, the wide is low and the narrow is high". In theory, when having a CT scan for the tissues and organs, the selected window width should cover all the data (CT value) scope of the tissues and organs (The actual window width is often a little bit wider or narrower, it depends); the selected window level should happen to be central value of the scope. This may be the most appropriate window option.

The so-called "the high is black, the low is white" is for the window level. As the CT operator who is familiar with the CT image knows that, the black image indicates that the window level selection is higher and should be lower down; the white image indicates that the window level selection is lower and should be heighten.

The so-called "the wide is low and the narrow is high" is for the window width. The low contrast ratio indicates that the window width is wider and should be set narrower; the high contrast ratio indicates that the window width is narrower and should be set wider.

The modulations of the window level and window width were all by programming and operating the golden section algorithm. The golden ratio is 0.61803398875 (accurate to the 2003 digit after the decimal point, here is the approximation), we use 0.618 as window level and $0.382(=1-0.618)$ as window width by searching segmentation arithmetic during programming.

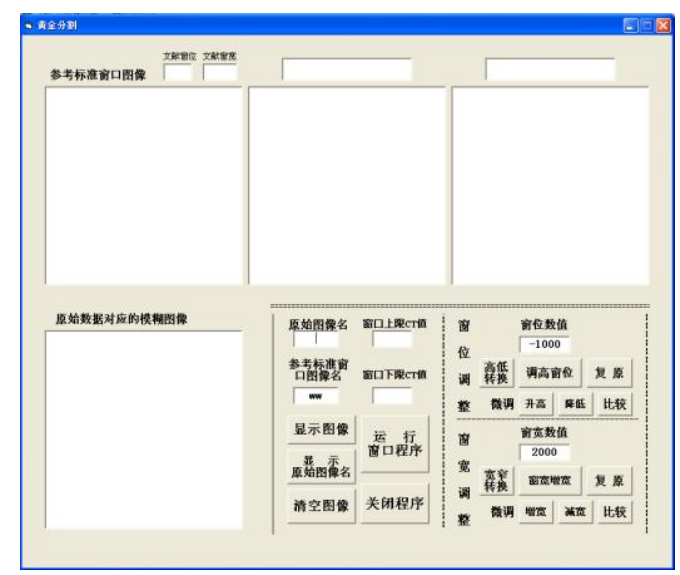

Figure 1 The program interface of finding out the right window by golden segmentation algorithm

Figure 1 is the program interface of finding out the right window by golden segmentation algorithm. As an example, the following is the process and outcome of quickly finding out the CT image by golden segmentation algorithm.

See figure 2, figure 2 is the corresponding blur CT image of the right window and the segmentation search progress of the window to the original data.
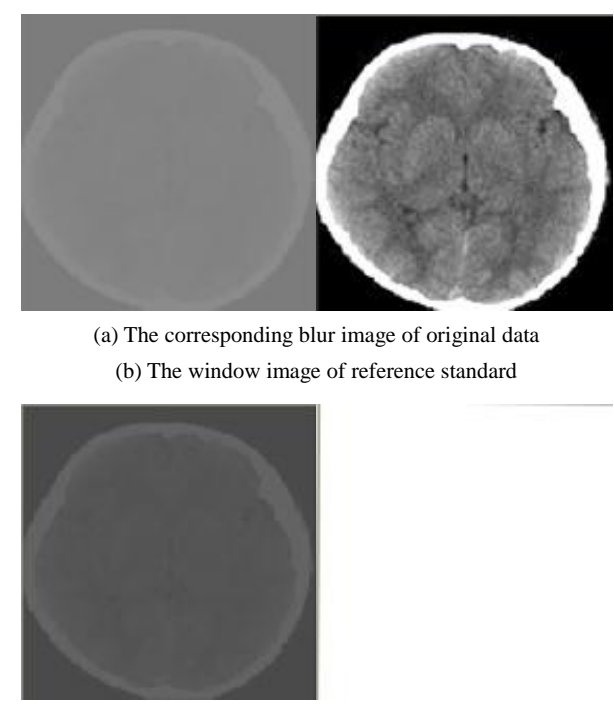

(c) The first split image

(d) The second split image (all white)

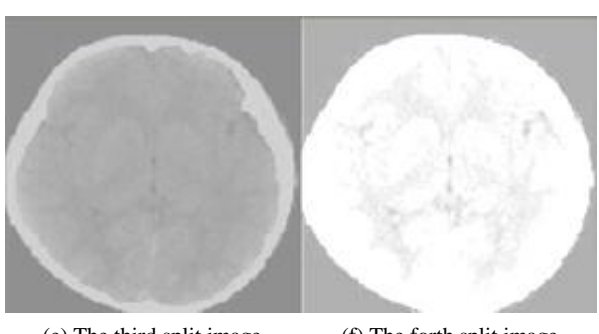

$\begin{array}{ll}\text { (e) The third split image } & \text { (f) The forth split image }\end{array}$ 


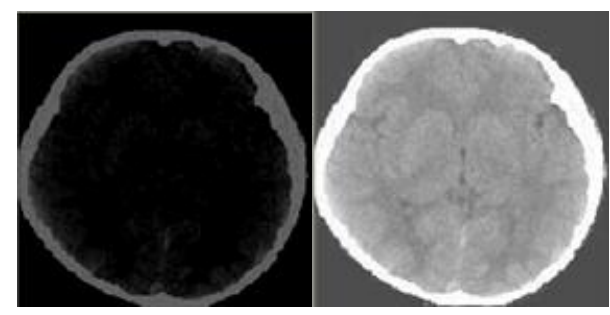

(g) The fifth split image

(h) The sixth split image

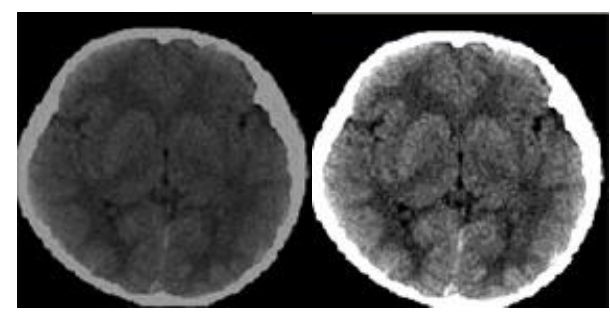

(i) The seventh split image

(j) The eighth split image

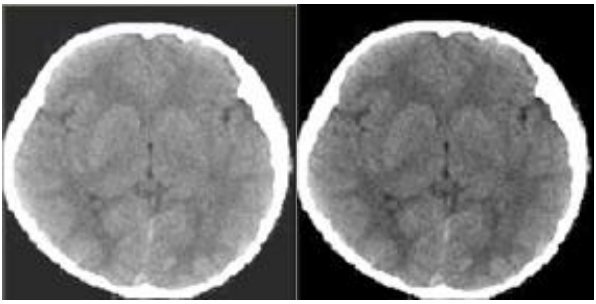

(k) The ninth split image

(l) The tenth split image

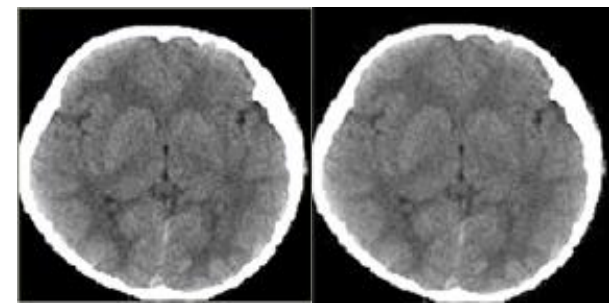

(m) The eleventh split image

(n) The twelfth split image

Figure 2 the windows of a blur CT image and the corresponding segmentation search progress

The first two images in figure 2 are the original blur image and the standard CT image as reference. Search for the right window by golden section program according to the criterion. The first split image is a little bit black, it indicates that the window level is high and should be lower down. The second image is the one after lower down the window level, which screen is all white, that indicates the window level is too low and should be heighten; the third image is the one after heighten the window level, the screen is still white and the contract ratio is low, suggesting that the window level remains low and the window width is wider, so the window level should be increased and the window width should be narrower; the forth image is the one after heightening the window level and narrowing the window width, but the screen is still white, suggesting that the window level remains low and should be heighten up; the fifth image is a little bit black after heightening up the window level, suggesting the window level is high and should be lower down; the picture of the sixth image is still white and with low contract ratio after lower down the window level, that indicates that the window level is low and window width is wider, so the window level should be higher and the window width should be narrower; the seventh image is the one after heightening up the window level, the image is black which indicates that the window level is high and should be lower down; after lowering down the window level, the contact ratio of the eighth image is high, suggesting the window width is narrower and should be wider; after widening the window width, the picture of the ninth image is white, suggesting that the window level is low and should be heighten up; the tenth image is the one after heightening up the window level, the image is black and with high contact ratio, suggesting that the window level is higher and the window width is narrow, so the window level should be lower down and the window width should be widen; after that, here is the eleventh image, its picture still remains black and the window level should be lower down; the twelfth segmentation image is the last image, the comparison of the twelfth image and the standard CT image shows that they are almost the same, the split operation works very well. If the black or white of the image and the high or the low of the contract ratio cannot be distinguished by naked eyes, the "comparison" procedure can be started. The above shows that to find out the right window and its corresponding image, we only tried 12 times. We can accomplish a split image in one second with skilled operation and accurate grasp of the criterion, the whole procedure only takes 20 seconds.

See figure 3 , figure 3 is the segmentation window search of a blur digital image.

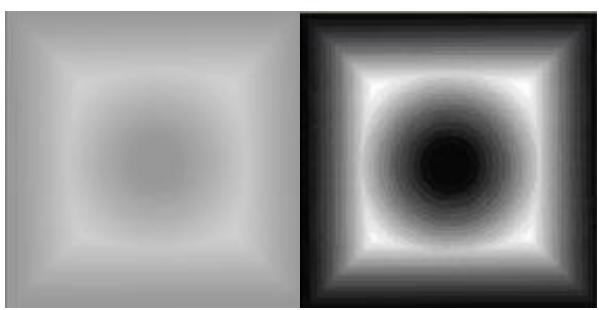

(a) The corresponding blur image of original data (b) The window image of reference standard

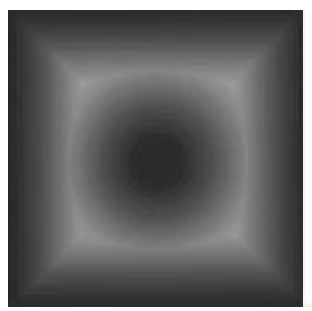

(c) The first split image (d) The second split image (all white)

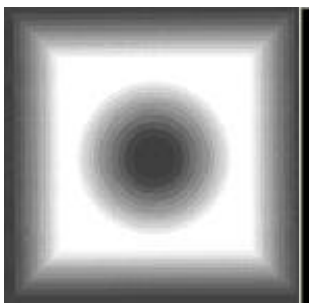

(e) The third split image

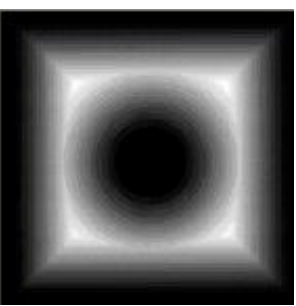

(f) The forth split image 


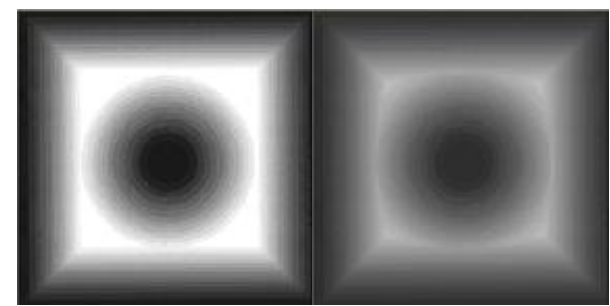

(g) The fifth split image

(h) The sixth split image

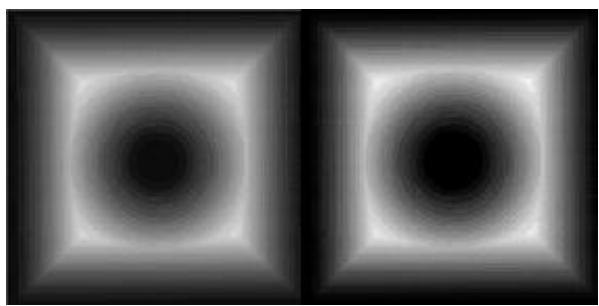

(i) The seventh split image

(j) The eighth split image

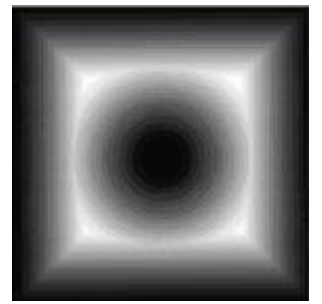

(k) The ninth split image

Figure 3 the segmentation window search of a blur digital image.

To find out the right window and its corresponding image to that blur digital image, we only tried nine times (the second, the third and the fifth split images are lack of image structures and lose the image information because of the inappropriate window). The last image in figure 3 is the eventual clear image after skilled and correct operation. The whole procedure takes only more than ten seconds.

\section{Conclusions}

The above described that the golden segmentation algorithm applied to the window search of CT image is faster and better compared to the double-window technique and multiple-window technique. Therefore, we consider that the golden segmentation algorithm has certain advantages when applying to the optimal window, is a very good window technology with practical value. This technique can be complement of CT window techniques and can provide reference for CT suppliers.

\section{References}

[1] Sun weihong, Flexible application of window technique used in CT examination, Journal of Practical Medical Techniques, 2010, 17(4):339-340.

[2] Feng Min, Yin Shanshan. Medical image processing based on outlier analysis, Journal of Clinical Rehabilitative Tissue Engineering Research, 2011, 15(39):7340-7342.

[3] Li Jingyu, Mu Weibin, Jin Cheng, Geng Kui, Zhang Yan, Research of image segmentation technique applied in medical image processing, Image Processing and Multimedia Technology, 2012, 31 (8):29-31.

[4] Wang Xiaobing, Sun Junyun, Applications of Several Segmentation Technologies in Medical Image Processing, Chinese Medical Equipment Journal, 2011, 32(7):76-78, 81.

[5] Gai Liping, Wang Li, Sun Bofu, The Displayed images of Different Scanning and Processing Methods for Medical Images. (2012-12). http://www.cnki.com.cn/Article/CJFDTotal-YNWS201212026.htm.

[6] Jia Hong, Zhang Jing, Gao Dengfa, The Effects on Display of DR Image Details after Different Treatments. (2010-01).http://www.cnki.com.cn/Article/CJFDTotal-ZYXX20100108 6.htm.

[7] Zhang Zebao, Ji Qang, Hu Jiguang, Medical Imaging Physics. Second Edition. People's Medical Publishing House, 2007:39-46. 